\title{
Candida albicans and Candida dubliniensis Show Different Trailing Effect Patterns When Exposed to Echinocandins and Azoles
}

\section{OPEN ACCESS}

Edited by:

Miguel Cacho Teixeira,

University of Lisbon, Portugal

Reviewed by:

Jesus Guinea,

Gregorio Marañón Hospital, Spain

Cheshta Sharma,

The University of Texas Health

Science Center at San Antonio,

United States

*Correspondence:

Eric Dannaou

eric.dannaoui@aphp.fr

Marie-Elisabeth Bougnoux bougnoux@pasteur.fr;

elisabeth.bougnoux-

andremont@pasteur.fr

${ }^{\dagger}$ These authors have contributed equally to this work

Specialty section:

This article was submitted to Antimicrobials, Resistance

and Chemotherapy,

a section of the journal

Frontiers in Microbiology

Received: 19 December 2019

Accepted: 20 May 2020

Published: 16 June 2020

Citation:

Ayadi $R$, Sitterlé $E$, d'Enfert $C$, Dannaoui $E$ and Bougnoux M-E

(2020) Candida albicans and Candida

dubliniensis Show Different Trailing

Effect Patterns When Exposed

to Echinocandins and Azoles.

Front. Microbiol. 11:1286.

doi: 10.3389/fmicb.2020.01286

\section{Rania Ayadi' ${ }^{1}$ Emilie Sitterlé2,3, Christophe d'Enfert ${ }^{3}$, Eric Dannaoui ${ }^{1,4 * \dagger}$ and Marie-Elisabeth Bougnoux ${ }^{2,3 *+}$}

1 Unité de Parasitologie-Mycologie, Service de Microbiologie, Faculté de Médecine, APHP, Hôpital Européen Georges Pompidou, Université Paris-Descartes, Paris, France, ${ }^{2}$ Unité de Parasitologie-Mycologie, Service de Microbiologie, Faculté de Médecine, APHP, Hôpital Necker Enfants-Malades, Université Paris-Descartes, Paris, France, ${ }^{3}$ Unité Biologie et Pathogénicité Fongiques, Institut Pasteur, USC 2019 INRA, Paris, France, ${ }^{4}$ Dynamyc Research Group, Paris Est Créteil University (UPEC, EnvA), Créteil, France

When Candida albicans and Candida dubliniensis isolates were tested for susceptibility to fluconazole and echinocandins using either EUCAST or Etest methods, differential patterns of growth were observed, independently of the methods used. For C. albicans, a trailing phenomenon (incomplete growth inhibition at supra-MICs) was observed with fluconazole in 90\% and 93.3\% for EUCAST and Etest, respectively, but not with echinocandins ( $<7 \%$ for EUCAST and 0\% for Etest). In contrast, for C. dubliniensis, a trailing phenomenon was very rarely observed with fluconazole $(20 \%$ for EUCAST and $0 \%$ for Etest), while the opposite pattern was observed with echinocandins (>50\% for EUCAST and $>86 \%$ for Etest). This suggests that the pathways involved in the trailing effect might be different between these two related species. Furthermore, clinical microbiologists must be aware of these species-specific patterns for a reliable MIC determination.

Keywords: C. albicans, C. dubliniensis, trailing, EUCAST, antifungal susceptibility testing, Etest, echinocandins

\section{INTRODUCTION}

Candida dubliniensis is a commensal of the oral cavity but can also colonize other anatomical sites (Loreto et al., 2010). Furthermore, it can cause oropharyngeal candidiasis, mainly in HIV-positive patients (Sullivan et al., 2005; Loreto et al., 2010), and invasive candidiasis (Pfaller et al., 2014a). C. dubliniensis is closely related to Candida albicans (Sullivan et al., 2005). C. albicans and C. dubliniensis share the same in vitro antifungal susceptibility patterns (Pfaller et al., 1999; Lortholary et al., 2007). The clinical breakpoints (CBs) and Epidemiological Cut-Off values (ECOFFs), determined by CLSI/EUCAST reference methods or commercial methods are similar (Pfaller et al., 2014b; Espinel-Ingroff et al., 201919). Acquired resistance to antifungal has been reported in C. dubliniensis just as in C. albicans (Perea et al., 2002; Coleman et al., 2010; Prigent et al., 2017), but less frequently (Pfaller et al., 1999). Until recently, identification of C. dubliniensis was difficult in the clinical laboratories because it shares C. albicans phenotypic characteristics (Sullivan et al., 2005; Loreto et al., 2010). Things changed with the use of Matrix-Assisted Laser Desorption Ionization-Time of

\footnotetext{
${ }^{1}$ http://www.eucast.org/astoffungi/clinicalbreakpointsforantifungals/
} 
Flight (MALDI-TOF), which can easily differentiate the two species (Roberts et al., 2016). There are some striking differences between C. albicans and C. dubliniensis. First, their ecological niches are different. Unlike C. albicans, C. dubliniensis is uncommon in the gastro-intestinal tract of healthy individuals, but for the oral cavity (Strati et al., 2016). Second, there are major difference in term of pathogenicity, C. albicans being more pathogenic (Moran et al., 2012).
Recently, we also observed differential growth patterns between C. albicans and C. dubliniensis when they are tested by Etest for susceptibility to fluconazole and echinocandins. With C. dubliniensis, a trailing effect (incomplete growth inhibition at supra-MICs) was observed with echinocandins but not with fluconazole, while for $C$. albicans the opposite pattern was seen. To further assess these differences between the two species,

TABLE 1 | MIC distribution of fluconazole, caspofungin and micafungin against C. albicans and C. dubliniensis by EUCAST and Etest methods.

\begin{tabular}{|c|c|c|c|c|c|c|c|c|c|c|c|c|c|}
\hline \multicolumn{14}{|c|}{ Antifungal } \\
\hline \multirow[b]{2}{*}{ Species } & & & \multirow[b]{2}{*}{ ATF } & \multicolumn{10}{|c|}{ Concentration (ug/ml) } \\
\hline & \multicolumn{2}{|c|}{ Method } & & 0.008 & 0.015 & 0.03 & 0.06 & 0.125 & 0.25 & 0.5 & 1 & 2 & 4 \\
\hline C. albicans & EUCAST & $24 \mathrm{~h}$ & FCZ & & & & & 14 & 14 & 2 & & & \\
\hline C. dubliniensis & & & & & & & & 23 & 5 & 1 & & 1 & \\
\hline C. albicans & EUCAST & $24 \mathrm{~h}$ & CAS & & & & & 8 & 21 & 1 & & & \\
\hline C. dubliniensis & & & & & & & & 8 & 16 & 6 & & & \\
\hline C. albicans & EUCAST & $24 \mathrm{~h}$ & MIC & & 30 & & & & & & & & \\
\hline C. dubliniensis & & & & & 30 & & & & & & & & \\
\hline C. albicans & EUCAST & $48 \mathrm{~h}$ & $\mathrm{FCZ}$ & & & & & 13 & 17 & & & & \\
\hline C. dubliniensis & & & & & & & & 22 & 4 & 1 & & 1 & \\
\hline C. albicans & EUCAST & $48 \mathrm{~h}$ & CAS & & & & & 5 & 21 & 4 & & & \\
\hline C. dubliniensis & & & & & & & & 6 & 11 & 12 & 1 & & \\
\hline C. albicans & EUCAST & $48 \mathrm{~h}$ & MIC & & 26 & 3 & 1 & & & & & & \\
\hline C. dubliniensis & & & & & 26 & 3 & 1 & & & & & & \\
\hline C. albicans & Etest & $24 \mathrm{~h}$ & FCZ & & & & 2 & 20 & 8 & & & & \\
\hline C. dubliniensis & & & & & & 2 & 13 & 12 & 2 & 1 & & & \\
\hline C. albicans & Etest & $24 \mathrm{~h}$ & CAS & & & 3 & 9 & 17 & 1 & & & & \\
\hline C. dubliniensis & & & & & & 1 & 3 & 24 & 2 & & & & \\
\hline C. albicans & Etest & $24 \mathrm{~h}$ & MIC & 25 & 5 & & & & & & & & \\
\hline C. dubliniensis & & & & 15 & 15 & & & & & & & & \\
\hline C. albicans & Etest & $48 \mathrm{~h}$ & FCZ & & & & 2 & 15 & 13 & & & & \\
\hline C. dubliniensis & & & & & & 1 & 13 & 13 & 1 & 2 & & & \\
\hline C. albicans & Etest & $48 \mathrm{~h}$ & CAS & & & & 9 & 15 & 6 & & & & \\
\hline C. dubliniensis & & & & & & & 3 & 18 & 9 & & & & \\
\hline C. albicans & Etest & $48 \mathrm{~h}$ & MIC & 20 & 10 & & & & & & & & \\
\hline C. dubliniensis & & & & 6 & 21 & 3 & & & & & & & \\
\hline
\end{tabular}

TABLE 2 | Trailing observed for fluconazole, micafungin, and caspofungin against C. albicans and C. dubliniensis by EUCAST and Etest methods.

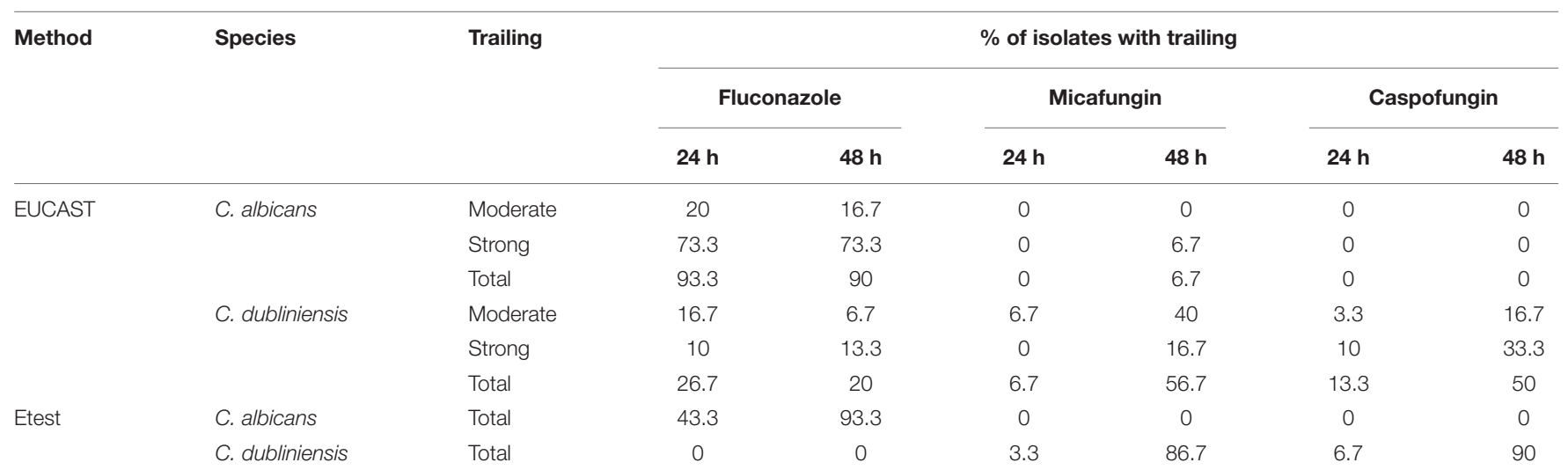



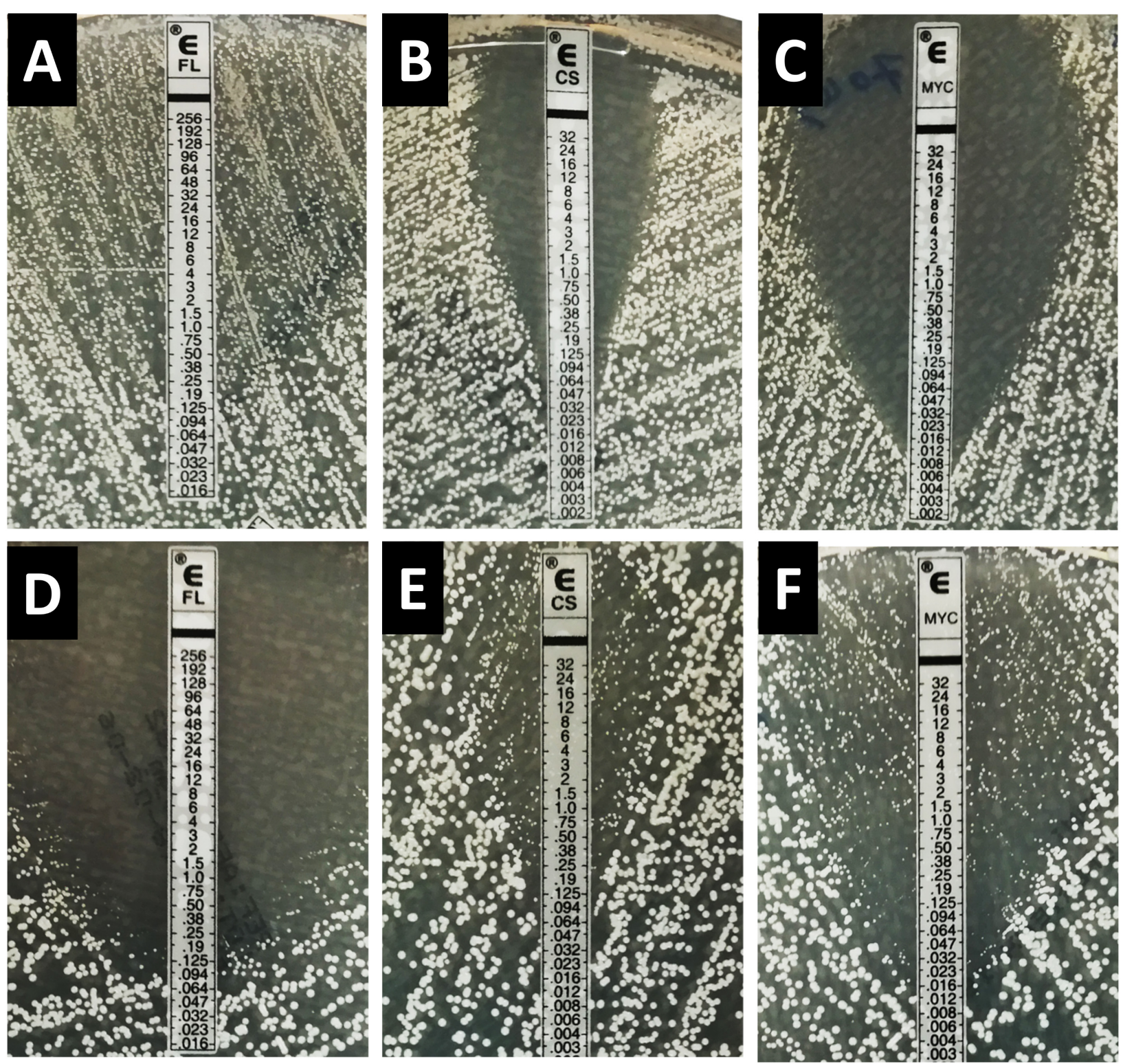

FIGURE 1 | Inhibition pattern by Etest for fluconazole, caspofungin, and micafungin for the strain C. albicans HEGP 7043 (A-C) and C. dubliniensis HEGP 6443 (D-F). FL, fluconazole; CS, caspofungin; MYC, micafungin.

we analyzed, here, the growing patterns of a large panel of clinical isolates of the two species when tested against fluconazole and two echinocandins by the reference EUCAST technique and by Etest.

\section{MATERIALS AND METHODS}

To study the growth of C. albicans and C. dubliniensis in the presence of fluconazole, caspofungin or micafungin, a total number of $30 \mathrm{C}$. albicans and $30 \mathrm{C}$. dubliniensis clinical epidemiologically not related isolates were selected from the mycology's laboratories of Necker and Georges Pompidou
University hospitals. None of these strains were known to be resistant to echinocandins or azoles.

These 60 isolates were tested using two different methods: the EUCAST EDef 7.3 microdilution procedure and the Etest agar diffusion method (Biomérieux, Marcy-l'Étoile, France). The yeasts were first cultured on BBL CHROMagar Candida plate (Becton Dickinson GmbH, Heidelberg, France) for primary identification and then identified by MALDI-TOF spectrometry (microflex LT, Bruker Daltonik GmbH, Germany).

Antifungal susceptibility testing by EUCAST was performed according to the reference method (Arendrup et al., 2017) in RPMI 1640 medium buffered to $\mathrm{pH} 7.0$ with morpholinopropanesulfonic acid (MOPS) and supplemented with glucose to 
a final concentration of $2 \%$. The final inoculum was $0.5-2.510^{5}$ CFU/ml. Candida krusei ATCC 6258 and Candida parapsilosis ATCC 22019 isolates were used as quality control strains. Spectrophotometric reading was performed after incubation at $35^{\circ} \mathrm{C}$, and MICs were determined with a $50 \%$ inhibition endpoint. The percentage of growth was determined compared to the drug-free control. The trailing effect was defined as a reduced but persistent $(\geq 5 \%)$ growth $(\mathrm{G})$ above the MIC of the isolate, at least in four consecutive wells. Consequently, two groups of trailing effect were defined depending on $G$, as previously described (Rueda et al., 2017): moderate trailing for $G$ between 5 and $25 \%$, strong trailing when $\mathrm{G}$ is $>25$ but $<50 \%$,

Antifungal susceptibility testing by Etest was performed according to manufacturer's instructions (Biomérieux, 2013). The presence of microcolonies in the ellipse of inhibition after incubation at $35^{\circ} \mathrm{C}$ defined the trailing effect and was not indicative of resistance. Due to the subjective nature of the visual reading, the level of the trailing effect was not categorized by the Etest method.

For both techniques (EUCAST, 2019) reading was performed at 24 and $48 \mathrm{~h}$. Indeed, for Etest the period incubation depends on the growth and could be $24 \mathrm{~h}$ and/or $48 \mathrm{~h}$. Moreover, it is recommended to read at $48 \mathrm{~h}$ for detection of some phenomenon such as heteroresistance. For these reasons, Etest reading is often performed both at 24 and $48 \mathrm{~h}$. Therefore, we also determined MICs by EUCAST at $24 \mathrm{~h}$ and $48 \mathrm{~h}$.

\section{RESULTS}

With Etest, MIC values at $24 \mathrm{~h}$ determined for fluconazole, micafungin, and caspofungin for C. albicans ranged from 0.06 to $0.25 \mu \mathrm{g} / \mathrm{ml}$ [Geometric mean $(\mathrm{GM})=0.14 \mu \mathrm{g} / \mathrm{ml}$ ], from 0.008 to $0.016(\mathrm{GM}=0.009 \mu \mathrm{g} / \mathrm{ml})$, and from 0.03 to $0.25 \mu \mathrm{g} / \mathrm{ml}$ ( $\mathrm{GM}=0.09 \mu \mathrm{g} / \mathrm{ml})$, respectively, while for C. dubliniensis MICs ranged from 0.03 to $0.5(\mathrm{GM}=0.09 \mu \mathrm{g} / \mathrm{ml})$, from 0.008 to 0.016 $(\mathrm{GM}=0.01 \mu \mathrm{g} / \mathrm{ml})$, and from 0.03 to $0.25(\mathrm{GM}=0.12 \mu \mathrm{g} / \mathrm{ml})$, respectively. MIC distributions determined by Etest at both $24 \mathrm{~h}$ and $48 \mathrm{~h}$ are presented in Table 1.

With fluconazole, a trailing effect was observed with Etest for $93.3 \%$ of C. albicans isolates while no trailing was seen with the two echinocandins (Table 2 and Figure 1). In contrast, for the C. dubliniensis isolates, trailing was not observed with fluconazole, while trailing was observed for echinocandins in $86.7 \%$ and $90 \%$ of the isolates for micafungin and caspofungin, respectively (Table 2 and Figure 1). In order to confirm that the differential pattern of growth was not related to the susceptibility testing method used, all the strains were also tested by EUCAST (Table 1). MICs determined by EUCAST were within $\pm 2 \log _{2}$ dilutions of the Etest MICs in $>98 \%$ of the cases.

The inhibition patterns obtained with Etest, were also observed by EUCAST for C. albicans and C. dubliniensis (Table 2). Indeed, for fluconazole, the trailing was seen for C. albicans and not for C. dubliniensis while the opposite pattern was observed with echinocandins. For C. albicans, $90 \%$ of the isolates exhibited a trailing effect for fluconazole while only $6.7 \%$, and $0 \%$ of the isolates showed a trailing for micafungin, and

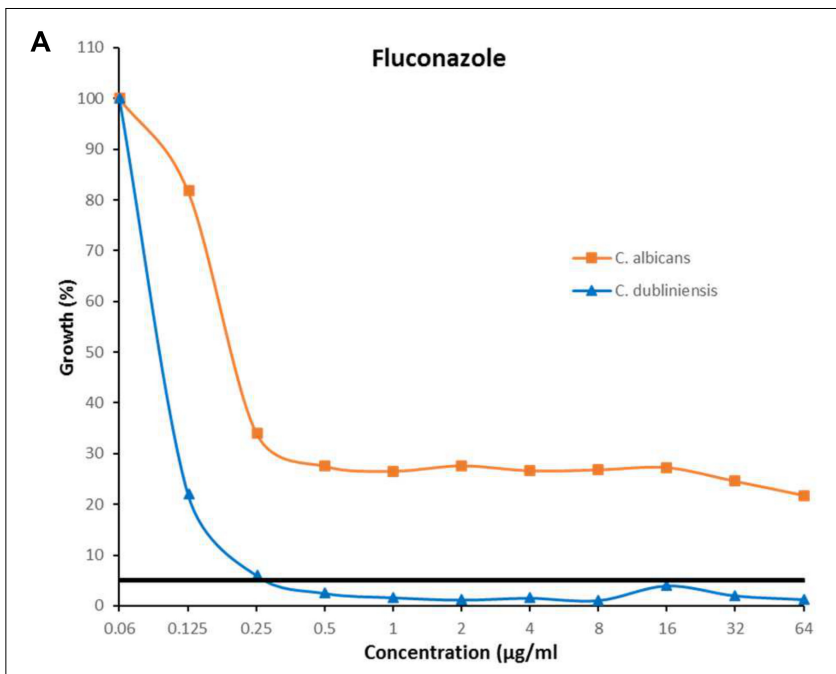

B

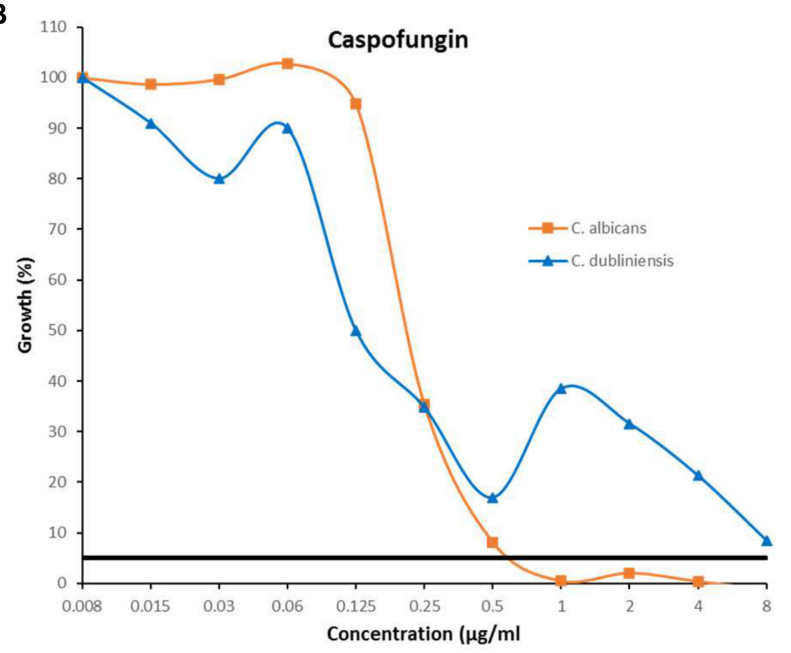

C

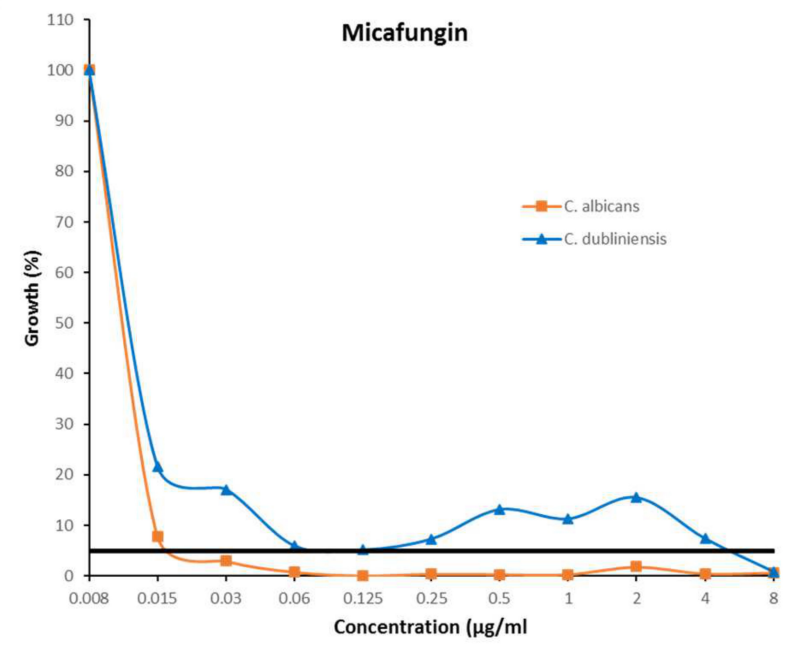

FIGURE 2 | Inhibition pattern by EUCAST in presence of fluconazole (A), caspofungin (B), and micafungin (C) for the strains C. albicans HEGP 7043 and C. dubliniensis HEGP 6409. MICs correspond to the lowest antifungal concentration for which the growth is $<50 \%$. Trailing correspond to a growth between 5 and $49 \%$. Black horizontal line represents the $5 \%$ growth threshold. 
caspofungin, respectively. For C. dubliniensis only $20 \%$ of the isolates showed a trailing effect for fluconazole, while $56.7 \%$, and $50 \%$ of isolates exhibited a trailing effect for micafungin and caspofungin, respectively (Table 2). All together, these data clearly demonstrated that the trailing is species specific. Figure 2 showed an example of the trailing effect observed for one isolate each of $C$. albicans and $C$. dubliniensis for the three antifungal agents.

The level of trailing effect was always of strong intensity (according to the definition) except for micafungin with C. dubliniensis isolates for which a moderate trailing was mostly observed (Table 2).

\section{DISCUSSION}

Although C. albicans and C. dubliniensis are closely related phylogenetically (Sullivan et al., 2005) and exhibit the same antifungal susceptibility patterns, we found that they behave differently in vitro in presence of fluconazole or echinocandins. For C. albicans, a trailing phenomenon was observed with fluconazole and not for the echinocandin, while for C. dubliniensis, the opposite pattern of growth was seen (i.e., no trailing with fluconazole and trailing with echinocandins). Differential in vitro response to echinocandin and azole antifungal agents between $C$. albicans and C. dubliniensis has been previously reported by EUCAST and CLSI methods (Arthington-Skaggs et al., 2002; Jacobsen et al., 2007). Our results are in accordance with these previous reports and show that the phenomenon is also present when isolates are tested

\section{REFERENCES}

Arendrup, M. C., Meletiadis, J., Mouton, J. W., Lagrou, K., Hamal, P., and Guinea, J. (2017). Subcommittee on Antifungal Susceptibility Testing (Afst) of the Escmid European Committee for Antimicrobial Susceptibility Testing (Eucast) in Method for the Determination of Broth Dilution Minimum Inhibitory Concentrations of Antifungal Agents for Yeasts. EUCAST definitive document E.Def 7.3.1. Clin. Microbiol. Infect. 18, E246-E247.

Arthington-Skaggs, B. A., Lee-Yang, W., Ciblak, M. A., Frade, J. P., Brandt, M. E., Hajjeh, R. A., et al. (2002). Comparison of visual and spectrophotometric methods of broth microdilution MIC end point determination and evaluation of a sterol quantitation method for in vitro susceptibility testing of fluconazole and itraconazole against trailing and nontrailing Candida isolates. Antimicrob. Agents Chemother. 46, 2477-2481. doi: 10.1128/aac.46.8.2477-2481.2002

Biomérieux, S. A. (2013). Etest Antifungal Susceptibility Testing Package Insert. France: bioMérieux SA,Marcy-l'Etoile.

Coleman, D. C., Moran, G. P., Mcmanus, B. A., and Sullivan, D. J. (2010). Mechanisms of antifungal drug resistance in Candida dubliniensis. Future Microbiol. 5, 935-949. doi: 10.2217/fmb.10.51

Delarze, E., and Sanglard, D. (2015). Defining the frontiers between antifungal resistance, tolerance and the concept of persistence. Drug Resist. Updat. 23, 12-19. doi: 10.1016/j.drup.2015.10.001

Espinel-Ingroff, A., Turnidge, J., Alastruey-Izquierdo, A., Botterel, F., Canton, E., Castro, C., et al. (2019). Method-dependent epidemiological cutoff values for detection of triazole resistance in Candida and Aspergillus species for the Sensititre YeastOne colorimetric broth and Etest agar diffusion methods. Antimicrob. Agents Chemother. 63:e001651-18.

EUCAST (2019). Subcommittee on Antifungal Susceptibility Testing (Afst) of the Escmid European Committee for Antimicrobial Susceptibility Testing. Available by a completely different method (i.e., Etest, an agar diffusion method), therefore not dependent on the technique.

Trailing effect of $C$. albicans to fluconazole is well known (Delarze and Sanglard, 2015; Marcos-Zambrano et al., 2016; Rosenberg et al., 2018) and may have a clinical impact (Rosenberg et al., 2018). Here we observed that this effect is not present for the sibling species C. dubliniensis. Altogether, these findings could suggest that the pathways involved in the trailing effect may be different in the two species. The comparative study of these two species may help deciphering the mechanisms involved in tolerance in C. albicans.

Finally, our findings are important for the clinical microbiology laboratories and clinical care. Indeed, the trailing observed when echinocandins are tested against $C$. dubliniensis should be known and the corresponding strains should not be considered as resistant pattern to these important drugs.

\section{DATA AVAILABILITY STATEMENT}

All datasets generated for this study are included in the article/supplementary material.

\section{AUTHOR CONTRIBUTIONS}

M-EB, CD'E, and ED designed the study. RA and ES performed the experiments. M-EB, RA, and ED analyzed the data. M-EB and ED wrote the manuscript. All authors listed have approved and corrected the manuscript.

online at: http://www.eucast.org/astoffungi/clinicalbreakpointsforantifungals/ (accessed July 16, 2019).

Jacobsen, M. D., Whyte, J. A., and Odds, F. C. (2007). Candida albicans and Candida dubliniensis respond differently to echinocandin antifungal agents in vitro. Antimicrob. Agents Chemother. 51, 1882-1884. doi: 10.1128/aac. 01525-06

Loreto, E. S., Scheid, L. A., Nogueira, C. W., Zeni, G., Santurio, J. M., and Alves, S. H. (2010). Candida dubliniensis: epidemiology and phenotypic methods for identification. Mycopathologia 169, 431-443. doi: 10.1007/s11046-010-9286-5

Lortholary, O., Dannaoui, E., Raoux, D., Hoinard, D., Datry, A., Paugam, A., et al. (2007). In vitro susceptibility to posaconazole of 1,903 yeast isolates recovered in France from 2003 to 2006 and tested by the method of the European Committee on Antimicrobial Susceptibility Testing. Antimicrob. Agents Chemother. 51, 3378-3380. doi: 10.1128/aac.00496-07

Marcos-Zambrano, L. J., Escribano, P., Sanchez-Carrillo, C., Bouza, E., and Guinea, J. (2016). Scope and frequency of fluconazole trailing assessed using EUCAST in invasive Candida spp. isolates. Med. Mycol. 54, 733-739. doi: 10.1093/mmy/ myw033

Moran, G. P., Coleman, D. C., and Sullivan, D. J. (2012). Candida albicans versus Candida dubliniensis: why is C. albicans more pathogenic?. Int. J. Microbiol. 2012:205921.

Perea, S., Lopez-Ribot, J. L., Wickes, B. L., Kirkpatrick, W. R., Dib, O. P., Bachmann, S. P., et al. (2002). Molecular mechanisms of fluconazole resistance in Candida dubliniensis isolates from human immunodeficiency virus-infected patients with oropharyngeal candidiasis. Antimicrob. Agents Chemother. 46, 1695-1703. doi: 10.1128/aac.46.6.1695-1703.2002

Pfaller, M. A., Andes, D. R., Diekema, D. J., Horn, D. L., Reboli, A. C., Rotstein, C., et al. (2014a). Epidemiology and outcomes of invasive candidiasis due to non-albicans species of Candida in 2,496 patients: data from the Prospective 
Antifungal Therapy (PATH) registry 2004-2008. PLoS One 9:e101510. doi: 10. 1371/journal.pone.0101510

Pfaller, M. A., Espinel-Ingroff, A., Bustamante, B., Canton, E., Diekema, D. J., Fothergill, A., et al. (2014b). Multicenter study of anidulafungin and micafungin MIC distributions and epidemiological cutoff values for eight Candida species and the CLSI M27-A3 broth microdilution method. Antimicrob. Agents Chemother. 58, 916-922. doi: 10.1128/aac.02020-13

Pfaller, M. A., Messer, S. A., Gee, S., Joly, S., Pujol, C., Sullivan, D. J., et al. (1999). In vitro susceptibilities of Candida dubliniensis isolates tested against the new triazole and echinocandin antifungal agents. J. Clin. Microbiol. 37, 870-872. doi: 10.1128/jcm.37.3.870-872.1999

Prigent, G., Ait-Ammar, N., Levesque, E., Fekkar, A., Costa, J. M., El Anbassi, S., et al. (2017). Echinocandin resistance in Candida species isolates from liver transplant recipients. Antimicrob. Agents Chemother. 61:e001229-16.

Roberts, A. L., Alelew, A., and Iwen, P. C. (2016). Evaluation of matrix-assisted laser desorption ionization-time-of-flight mass spectrometry to differentiate between Candida albicans and Candida dubliniensis. Diagn. Microbiol. Infect. Dis. 85, 73-76. doi: 10.1016/j.diagmicrobio.2016.01.017

Rosenberg, A., Ene, I. V., Bibi, M., Zakin, S., Segal, E. S., Ziv, N., et al. (2018). Antifungal tolerance is a subpopulation effect distinct from resistance and is associated with persistent candidemia. Nat. Commun. 9:2470.

Rueda, C., Puig-Asensio, M., Guinea, J., Almirante, B., Cuenca-Estrella, M., Zaragoza, O., et al. (2017). Evaluation of the possible influence of trailing and paradoxical effects on the clinical outcome of patients with candidemia. Clin. Microbiol. Infect. 23, e41-e49.
Strati, F., Di Paola, M., Stefanini, I., Albanese, D., Rizzetto, L., Lionetti, P., et al. (2016). Age and gender affect the composition of fungal population of the human gastrointestinal tract. Front. Microbiol. 7:1227. doi: 10.3389/fmicb.2016. 01227

Sullivan, D. J., Moran, G. P., and Coleman, D. C. (2005). Candida dubliniensis: ten years on. FEMS Microbiol. Lett. 253, 9-17. doi: 10.1016/j.femsle.2005. 09.015

Disclosure: Outside the present work the authors have the following disclosures: During the past 5 years, ED has received research grants from MSD and Gilead; travel grants from Gilead, MSD, Pfizer, and Astellas, and speaker's fee from Gilead, MSD, and Astellas. M-EB has received research grants from MSD and Astellas; travel grants from Gilead, MSD, and Astellas, and speaker's fee from Gilead, MSD, and Astellas.

Conflict of Interest: The authors declare that the research was conducted in the absence of any commercial or financial relationships that could be construed as a potential conflict of interest.

Copyright (c) 2020 Ayadi, Sitterlé, d'Enfert, Dannaoui and Bougnoux. This is an open-access article distributed under the terms of the Creative Commons Attribution License (CC BY). The use, distribution or reproduction in other forums is permitted, provided the original author(s) and the copyright owner(s) are credited and that the original publication in this journal is cited, in accordance with accepted academic practice. No use, distribution or reproduction is permitted which does not comply with these terms. 